\title{
Influence of Oxygen Concentration on Resistance Switching Characteristics of Gallium Oxide
}

\author{
Jheng-Jie Huang, Ting-Chang Chang, Jyun-Bao Yang, Shih-Ching Chen, Po-Chun Yang, \\ Yu-Ting Chen, Hsueh-Chih Tseng, Simon M. Sze, Ann-Kuo Chu, and Ming-Jinn Tsai
}

\begin{abstract}
In this letter, we fabricated resistance random access memory in a $\mathrm{Pt} / \mathrm{GaOx} / \mathrm{TiN}$ structure with a bipolar resistance switching characteristic and a bistable resistance ratio of about two orders by $I-V$ sweeping. In order to increase the oxygen ion quantity in the gallium oxide layer, the proposed sample was sputtered in a mixed ambient of Ar and oxygen, and the resistance ratio was enhanced by 2.5 orders. In addition to the resistance ratio, set voltage distribution statistics show that the stability of gallium oxide sputtered in mixed Ar and oxygen gas was better than standard Ar-only sample.
\end{abstract}

Index Terms-Gallium oxide, nonvolatile resistance switching memory, resistance random access memory (RRAM).

\section{INTRODUCTION}

W ITH the development of portable electronic products, nonvolatile memory has been widely applied. However, traditional nonvolatile floating-gate memory is confronting some physical limits as devices continuously scale down [1][4]. In order to solve this problem, it is necessary to develop other kinds of nonvolatile memory, such as ferroelectric random access memory (RAM), magnetoresistive RAM, phasechange RAM, and resistive RAM (RRAM) [5]-[8]. Among these nonvolatile memory devices, RRAM has the advantages of low operating consumption, high density (because of simple device structure), and fast switching speed (about several nanoseconds). A variety of materials is found to have resistive switching characteristics in the former studies. For instance, the perovskite oxides ( $\mathrm{Pr} 0.7 \mathrm{Ca} 0.3 \mathrm{MnO} 3$ and $\mathrm{SrZrO} 3)$ [9] or binary metal oxides (Al2O3, $\mathrm{HfO} 2, \mathrm{MnO}, \mathrm{ZnO}$, and IGZO) [8], [10]-[15] can change resistance by some specific operation

Manuscript received June 1, 2012; accepted June 21, 2012. Date of publication August 16, 2012; date of current version September 21, 2012. This work was supported by the National Science Council of the Republic of China under Contract NSC 100-2120-M-110-003. The review of this letter was arranged by Editor T. San.

J.-J. Huang, S.-C. Chen, and H.-C. Tseng are with the Department of Physics, National Sun Yat-Sen University, Kaohsiung 80424, Taiwan.

T.-C. Chang is with the Department of Physics, National Sun Yat-Sen University, Kaohsiung 804, Taiwan, and also with the Advanced Optoelectronics Technology Center,National Cheng Kung University, Tainan 701, Taiwan.

J.-B. Yang, P.-C. Yang, Y.-T. Chen, and A.-K. Chu are with the Department of Photonics, National Sun Yat-Sen University, Kaohsiung 80424, Taiwan.

S. M. Sze is with the Institute of Electronics, National Chiao Tung University, Hsin-Chu 300, Taiwan.

M.-J. Tsai is with the Electronics and Optoelectronics Research Laboratory, Industrial Technology Research Institute, Chutung, Hsinchu 310, Taiwan.

Color versions of one or more of the figures in this letter are available online at http://ieeexplore.ieee.org.

Digital Object Identifier 10.1109/LED.2012.2206365 condition. In previous research, one of the investigated properties of the gallium oxide is its luminescence, and it can be applied to the optoelectronic device. Another is the characteristic of oxygen sensation because its resistance value would be modified by the oxygen concentration of ambient environment [16], [17]. Additionally, the resistive switching mechanisms of the RRAM device are generally dominated by the migration of oxygen. Hence, we choose the gallium oxide as the material of the resistive switching layer to investigate the influence of oxygen concentration on the resistive switching characteristics. From the experiment results, the resistance value of the proposed device is actually sensitive to the oxygen concentration in the gallium oxide thin film.

In this letter, we investigate the oxygen concentration of a resistance switching layer because some literature has found that resistive switching characteristics are related to the movement of oxygen ions. We deposited gallium oxide films of two oxygen concentrations and analyzed the difference of their properties.

\section{EXPERIMENT}

The proposed RRAM device was fabricated on a TiN/SiO2/Si substrate with $\mathrm{SiO} 2$ and TiN thicknesses of 500 and $20 \mathrm{~nm}$, respectively. On the TiN layer, we deposited a 500-nm low-temperature silicon oxide layer and etched a contact hold to define the size of the device and exposed the TiN bottom electrode. First, the resistance switching layer of a 30-nm-thick gallium oxide film was deposited by radiofrequency sputtering a Ga0.4O0.6 target in a mixed ambient of $\mathrm{Ar}(30 \mathrm{sccm})$ and $\mathrm{O} 2(10 \mathrm{sccm})$ at room temperature. We also deposited a similar device with 30-nm-thick gallium oxide in $\operatorname{Ar}(30 \mathrm{sccm})$ ambient only as the control sample. Subsequently, we sputtered 100-nm-thick Pt on the gallium oxide as the top electrode, completing the RRAM device with a $\mathrm{Pt} / \mathrm{GaO} / \mathrm{TiN}$ structure. In order to investigate the resistive switching properties of the gallium oxide film, we measured the $I-V$ curves by an Agilent B1500 semiconductor parameter analyzer. In addition, the formation of gallium oxide film was analyzed by X-ray photoelectron spectroscopy (XPS, JEOL JPS 9010 MX).

\section{RESUlT AND Discussion}

To measure the characteristics of the $\mathrm{Pt} / \mathrm{GaO} / \mathrm{TiN}$ device, we applied voltage on the TiN electrode and grounded the Pt electrode, as shown in the inset in Fig. 1(a). First, we applied 

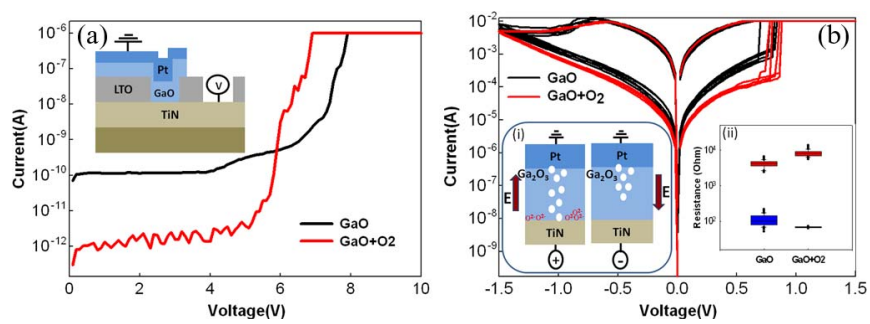

Fig. 1. RRAM characteristics of (a) forming process and (b) bipolar switching.

$+10 \mathrm{~V}$ on the TiN electrode with a compliance current of $1 \mu \mathrm{A}$, and the soft breakdown of the gallium oxide thin film was obtained, as shown in Fig. 1(a). Compared with the control sample, the gallium oxide with additional oxygen ambient had lower leakage current before the forming process. After the forming process, the leakage path forms by oxygen vacancies and the oxygen ions diffuse to the TiN. At this moment, the device is at low-resistance state. When a negative sweeping voltage (from 0 to $-1.6 \mathrm{~V}$ ) applied on TiN, the oxygen ions move away from the TiN and combine with the oxygen vacancies near the TiN. The leakage path ruptures, and the device switches to high-resistance state (HRS). A subsequent application of positive voltage sweeping from 0 to $2 \mathrm{~V}$ on the TiN electrode causes the leakage path of oxygen vacancies to reform [8], [12]-[14], [18], [19]. The inset (i) of Fig. 1(b) presents the set/reset process mechanism. This switching behavior can be repeated, and the ON/OFF resistance value was extracted at $0.2 \mathrm{~V}$. In order to extract the ON/OFF ratio and set voltage (resistive switching characteristics of gallium oxide), each device underwent dc cycle sweeps for three hundred times. Clearly, the oxygen-rich gallium oxide exhibits better bistable resistive switching properties, and the resistance value of HRS is higher than the control sample, as shown in inset (ii) of Fig. 1(b).

In order to investigate the formation of the insulator, the composition of the gallium oxide layer was analyzed by XPS. Fig. 2(a) shows the XPS analysis of O 1s core-level spectra for the gallium oxide film with different process ambient. The XPS data of oxygen show that the intensity of the gallium oxide film with additional oxygen is higher than the Arambient control sample. This result indicates that the additional oxygen atmosphere in the sputtering process can effectively increase the oxygen concentration of the gallium oxide film. Fig. 2(b) and (c) are the XPS analyses of Ga 3d5/2, using the Lorenzian-Gaussian functions to investigate the chemical states of the sample. By this analysis method, we separated out two peaks from the $\mathrm{Ga} 3 \mathrm{~d} 5 / 2$ spectra. One peak at the binding energy of $19.26 \mathrm{eV}$ is $\mathrm{Ga}-\mathrm{O}$ bonding, and the other peak at the $18.36 \mathrm{eV}$ is $\mathrm{Ga}-\mathrm{Ga}$ bonding. From the peak area present in the XPS analysis, we can verify the composition of the resistive switching layer. Compared with the control sample, the area of the $\mathrm{Ga}-\mathrm{O}$ peak of gallium oxide film with additional oxygen would increase, and the peak area of Ga-Ga would decrease. The additional oxygen serves to oxidize the $\mathrm{Ga}$ and to decrease the quantity of defects.

We analyzed the leakage current mechanism by the basic conduction process in insulators. Fig. 1(b) shows that the
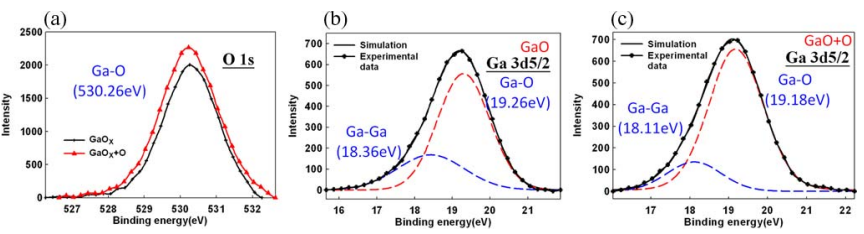

Fig. 2. XPS analysis of (a) O1s, (b) Ga3d5/2 of control sample, and (c) Ga3d5/2 of the gallium oxide film with additional oxygen. The dashed red line is $\mathrm{Ga}-\mathrm{O}$ bonding, and the dashed blue line is $\mathrm{Ga}-\mathrm{Ga}$ bonding.
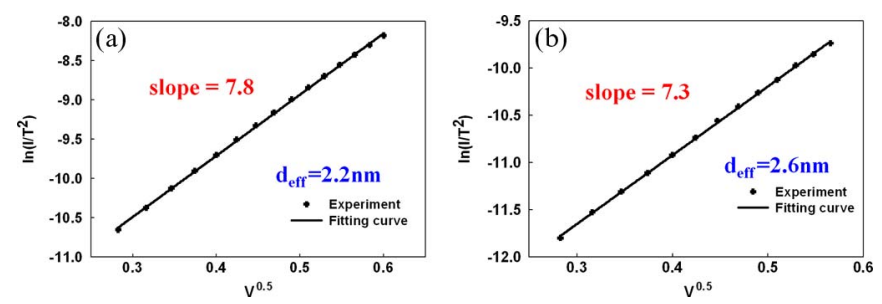

Fig. 3. Analysis of the leakage current in (a) control sample and (b) gallium oxide film with additional oxygen.

variation of HRS was influenced by oxygen concentration. In order to clarify how oxygen concentration affects the resistance value of HRS, we analyzed the $I-V$ curve. The analysis result indicates that the conduction process of HRS is thermionic emission (Schottky) current, as shown in Fig. 3. From the Schottky current equation, we have

$$
J=A T^{2} \exp \left[\frac{-q\left(\phi_{B}-\sqrt{q E / 4 \pi \varepsilon_{i}}\right)}{k T}\right] .
$$

We can find $\ln \left(I / T^{2}\right)$ versus $\sqrt{V}$ with the slope of equation being $q \sqrt{q / 4 \pi \varepsilon_{i} d_{\mathrm{eff}}} / k T$. The $\varepsilon_{i}$ (the permittivity of gallium oxide is 15.8), $T, k, A, \phi_{B}$, and $d_{\mathrm{eff}}$ are the permittivity, temperature, the Boltzmann constant, the effective Richardson constant, the barrier between electrode and insulator at zero voltage, and the effective thickness of the resistive switching layer, respectively. According to the $C-V$ curves of the $\mathrm{Pt} / \mathrm{GaO}$ $+\mathrm{O} / \mathrm{TiN}$ device, the capacitance is about $3.9 \times 10^{-13}$. Therefore, we can get the permittivity of gallium oxide $\left(\varepsilon_{i}\right)$ from the following:

$$
C=\varepsilon_{i} \frac{A}{d}=\varepsilon_{0} \varepsilon_{R} \frac{A}{d}
$$

where $\varepsilon_{0}$ is the permittivity in vacuum $\left(8.854 \times 10^{-14}\right), \varepsilon_{R}$ is the relative permittivity of gallium oxide, $A$ is the area $(8 \mu \mathrm{m} \times$ $8 \mu \mathrm{m})$, and $d$ is the thickness of the device $(23 \mathrm{~nm})$. Accordingly, the relative permittivity of gallium oxide is estimated to be about 15.8. Therefore, we can calculate the effective thickness of the resistive switching layer from the slope. From the slope value (7.8 for the control sample and 7.3 for the gallium oxide with additional oxygen), we can then estimate the thickness of $d_{\text {eff }}$ to be 2.2 and $2.6 \mathrm{~nm}$, respectively. This result suggests that the additional oxygen oxidizes many oxygen vacancies near the TiN layer such that the thickness of the switching layer will be thicker than the control Ar ambient sample.

Fig. 4(a) shows the distribution of set voltages under the same operation condition, with the data indicating that the variation of set voltages in gallium oxide with additional oxygen is smaller than in the control sample. With additional oxygen, the 

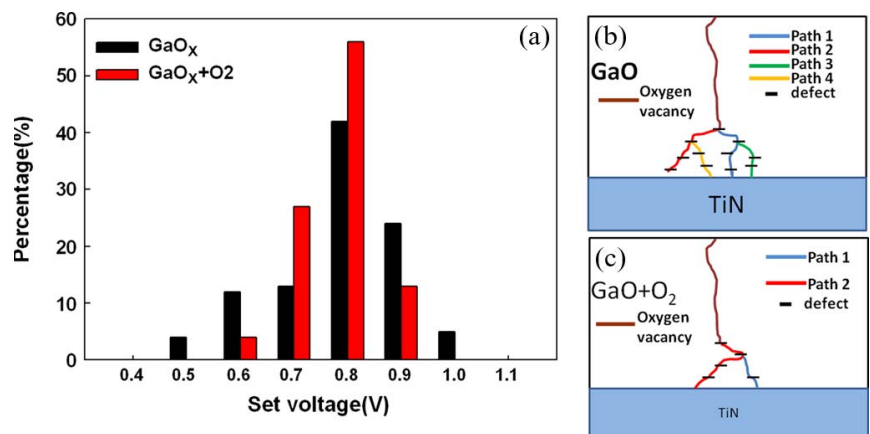

Fig. 4. (a) Distribution of set voltages under the same operation condition. Defects and leakage paths in (b) control sample and (c) gallium oxide film with additional oxygen.

instances of lower distributed (below 0.7) set voltages decrease, instead of converging on higher voltages. This is because the leakage path is dominated by the quantity of defects in the gallium oxide film, as shown in Fig. 4(b). The more defects that exist in the gallium oxide film, the more leakage paths will form during each set/reset cycle, with different leakage paths resulting in different set voltages. The additional oxygen acts to reduce the quantity of defects [19], thereby reducing the quantity of leakage paths, resulting in higher set voltages, as shown in Fig. 4(c).

\section{CONCLUSION}

In summary, we sputtered a gallium oxide layer in $\mathrm{Ar}+\mathrm{O} 2$ mixed ambient to enhance the concentration of oxygen atoms in the gallium oxide film. Because the additional oxygen acts to oxidize more vacancies, the resistance of HRS increases, and the thickness of the switching layer increases with the rise in oxygen concentration. The higher resistance (lower current) can reduce power consumption. Furthermore, the proposed method can decrease the distribution of set voltages and make the device more stable during the operation process.

\section{ACKNOWLEDGMENT}

The authors would like to thank the National Science Council Core Facilities Laboratory for Nanoscience and Nanotechnology in the Kaohsiung-Pingtung area, where the experiment for this work was performed.

\section{REFERENCES}

[1] D. Jiang, M. Zhang, Z. Huo, Q. Wang, J. Liu, Z. Yu, X. Yang, Y. Wang, B. Zhang, J. Chen, and M. Liu, "A study of cycling induced degradation mechanisms in Si nanocrystal memory devices," Nanotechnology, vol. 22, no. 25, pp. $254009-1-254$ 009-5, Jun. 2011.

[2] C. Chang, T. Yan, T. Liu, W. Chen, H. Lin, and M. Sze, "A novel approach of fabricating germanium nanocrystals for nonvolatile memory application," Electrochem. Solid State Lett., vol. 7, no. 1, pp. G17-G19, 2004.
[3] W. R. Chen, T. C. Chang, P. T. Liu, P. S. Lin, C. H. Tu, and C. Y Chang, "Formation of stacked $\mathrm{Ni}$ silicide nanocrystals for nonvolatile memory application," Appl. Phys. Lett., vol. 90, no. 11, pp. 112 108-1-112 108-3, Mar. 2007.

[4] T. C. Chang, F. Y. Jian, S. C. Chen, and Y. T. Tsai, "Developments in nanocrystal memory," Mater. Today 14, vol. 12, pp. 608-615, 2011.

[5] G. Asano, H. Morioka, and H. Funakubo, "Fatigue-free $\mathrm{RuO}_{2}$ / $\mathrm{Pb}(\mathrm{Zr}, \mathrm{Ti}) \mathrm{O}_{3} / \mathrm{RuO}_{2}$ capacitor prepared by metalorganic chemical vapor deposition at $395{ }^{\circ}$ C," Appl. Phys. Lett., vol. 83, no. 26, pp. 5506-5508, Dec. 2003.

[6] A. Ney and J. S. Harris, Jr., "Reconfigurable magnetologic computing using the spin flop switching of a magnetic random access memory cell," Appl. Phy. Lett., vol. 86, no. 1, pp. 013502-1-013502-3, Jan. 2004.

[7] T. C. Chong, L. P. Shi, R. Zhao, P. K. Tan, J. N. Li, H. K. Lee, X. S Miao, A. Y. Du, and C. H Tung, "Phase change random access memory cell with superlattice-like structure," Appl. Phys. Lett., vol. 88, no. 12, pp. 122 114-1-122 114-3, Mar. 2006.

[8] C. Y. Lin, C. Y. Wu, C. Y. Wu, C. Hu, and T. Y. Tseng, "Bistable resistive switching in $\mathrm{Al}_{2} \mathrm{O}_{3}$ memory thin films," J. Electrochem. Soc., vol. 154, no. 9, pp. G189-G192, Jul. 2007.

[9] C. Y. Liu, P. H. Wu, A. Wang, W. Y. Jang, J. C. Young, K. Y. Chiu, and T. Y. Tseng, "Bistable resistive switching of a sputter-deposited Cr-doped $\mathrm{SrZrO}_{3}$ memory film," IEEE Electron Device Lett., vol. 26, no. 6, pp. 351-353, Jun. 2005.

[10] Y. T. Tsai, T. C. Chang, C. C. Lin, S. C. Chen, C. W. Chen, S. M. Sze, F. S. Yeh (Hung), and T. Y. Tseng, "Influence of nanocrystals on resistive switching characteristic in binary metal oxides memory devices," Electrochem. Solid State Lett., vol. 14, no. 3, pp. H135-H138, 2011.

[11] H. Y. Lee, P. S. Chen, T. Y. Wu, C. C. Wang, P. J. Tzeng, C. H. Lin, F. Chen, M. J. Tsai, and C. Lien, "Electrical evidence of unstable anodic interface in Ru/HfO $/$ /TiN unipolar resistive memory," Appl. Phys. Lett., vol. 92, no. 14, pp. 142911-1-142 911-3, Apr. 2008.

[12] Q. Liu, S. Long, H. Lv, W. Wang, J. Niu, Z. Huo, J. Chen, and M. Liu, "Controllable growth of nanoscale conductive filaments in solid-electrolyte-based ReRAM by using metal nanocrystal cover bottom electrode," Appl. Phys. Lett., vol. 4, no. 10, pp. 6162-6168, Oct. 2010.

[13] N. Xu, L. Liu, X. Sun, X. Liu, D. Han, Y. Wang, R. Han, J. Kang, and $\mathrm{B}$. Yu, "Characteristics and mechanism of conduction/set process in TiN/ZnO/Pt resistance switching random-access memories," Appl. Phys. Lett., vol. 92, no. 23, pp. 232 112-1-232 112-3, Jun. 2008.

[14] M. C. Chen, T. C. Chang, C. T. Tsai, S. Y. Huang, S. C. Chen, C. W. Hu, S. M. Sze, and M. J. Tsai, "Influence of electrode material on the resistive memory switching property of indium gallium zinc oxide thin films," Appl. Phys. Lett., vol. 96, no. 26, pp. 262 110-1-262 110-3, Jun. 2010.

[15] M. C. Chen, T. C. Chang, S. Y. Huang, S. C. Chen, C. W. Hu, C. T. Tsai, and S. M. Sze, "Bipolar resistive switching characteristics of transparent indium gallium zinc oxide resistive random access memory," Electrochem. Solid State Lett., vol. 13, no. 6, pp. H191-H193, 2010

[16] M. Ogita, Y. Higo, Y. Nakanishi, and Y. Hatanaka, " $\mathrm{Ga}_{2} \mathrm{O}_{3}$ thin film for oxygen sensor at high temperature," Appl. Surf. Sci., vol. 175/176, pp. 721-725, May 2001.

[17] M. C. Quinlan, M. J. O'Donnell, L. Binet, and D. Gourier, "Origin of the blue luminescence of b-Ga $\mathrm{O}_{3}$," J. Phys. Chem. Solids, vol. 59, pp. 12411249, 1998.

[18] P. C. Yang, T. C. Chang, S. C. Chen, Y. S. Lin, H. C. Huang, and D. S. Gan, "Influence of bias-induced copper diffusion on the resistive switching characteristics of SiON thin film," Electrochem. Solid State Lett., vol. 14, no. 2, pp. H93-H95, 2011.

[19] X. Gao, Y. Xai, J. Ji, H. Xu, Y. Su, H. Li, C. Yang, H. Guo, J. Yin, and Z. Liu, "Effect of top electrode materials on bipolar resistive switching behavior of gallium oxide films," Appl. Phys. Lett., vol. 97, no. 19, pp. 193 501-1-193 501-3, Nov. 2010. 